\title{
Alterstice
}

Revue internationale de la recherche interculturelle

International Journal of Intercultural Research

Revista International de la Investigacion Intercultural

\section{Retour aux études : processus de (perte de) confiance et d'acculturation chez des étudiants immigrants du Campus Montréal [synthèse en français]}

\section{Sébastien Arcand, Chantale Asselin et Fasal Kanouté}

Volume 6, numéro 1, 2016

Prendre en compte la diversité à l'école

URI : https://id.erudit.org/iderudit/1038280ar

DOI : https://doi.org/10.7202/1038280ar

Aller au sommaire du numéro

Éditeur(s)

Alterstice

ISSN

1923-919X (numérique)

Découvrir la revue

Citer ce document

Arcand, S., Asselin, C. \& Kanouté, F. (2016). Retour aux études : processus de (perte de) confiance et d'acculturation chez des étudiants immigrants du Campus Montréal [synthèse en français]. Alterstice, 6(1), 73-75.

https://doi.org/10.7202/1038280ar 


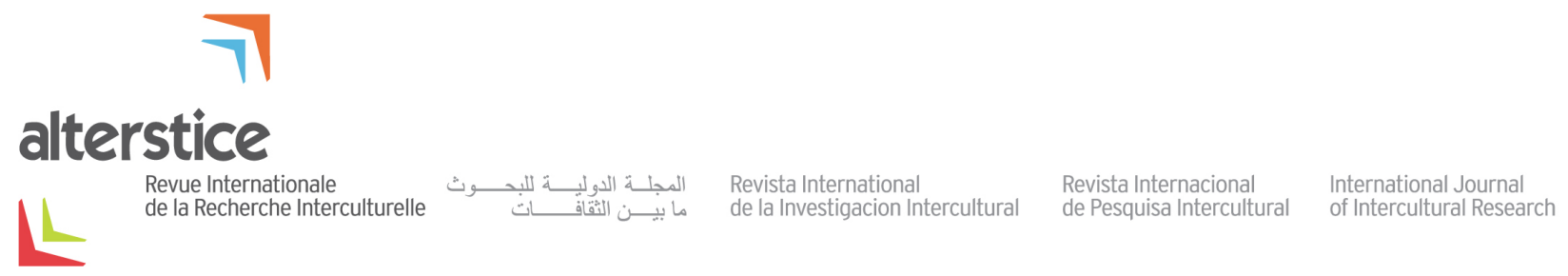

ARTICLE THÉMATIQUE

\title{
Retour aux études : processus de (perte de) confiance et d'acculturation chez des étudiants immigrants du Campus Montréal [synthèse en français*]
}

\author{
Sébastien Arcand ${ }^{1}$, Chantale Asselin ${ }^{2}$ et Fasal Kanouté ${ }^{3}$
}

\author{
Rattachement des auteurs \\ ${ }^{1}$ Département de management, HEC Montréal (Montréal), Canada ; ${ }^{2}$ chercheure indépendante ; ${ }^{3}$ Université de \\ Montréal, Montréal (Canada)
}

\section{Correspondance}

sebastien.arcand@hec.ca

\section{Mots clés}

academic and professional integration, acculturation, intergroup relations, trust, social representations

\section{Pour citer cet article}

Arcand, S., Asselin, C. et Kanouté F. (2016). Retour aux études : processus de (perte de) confiance et d'acculturation chez des étudiants immigrants du Campus Montréal [synthèse en français]. Alterstice, 6(1), 73-76. [Back to school! (Mis)trust and acculturation processes among immigrant students at Campus Montreal]

*L'article complet en anglais est disponible sur le site d'Alterstice.

Bon nombre de nouveaux arrivants se tournent vers les institutions scolaires et universitaires pour acquérir un diplôme délivré par une institution de leur société d'accueil et ainsi augmenter leur capital social sur le marché du travail. Malgré les nombreuses études sur ces populations issues de l'immigration au sein des différents systèmes scolaires des sociétés d'accueil, rares sont celles qui se sont intéressées à la catégorie des immigrants résidents permanents, un statut de transition vers l'acquisition de la citoyenneté (il permet de jouir de presque tous les avantages attribués à la citoyenneté sauf le droit de vote). II s'agit pourtant d'une catégorie intéressante car ce sont des personnes ayant de fortes chances de demeurer dans le pays d'accueil et de contribuer activement à son développement socio-économique (la plupart d'entre eux demandent leur citoyenneté en moins de 5 ans). Cet article porte sur des étudiants résidents permanents ayant intégré, au cours des dernières années, le système universitaire de la province de Québec (Canada).

Nous mettons ici l'accent sur la nature des relations qu'entretiennent ces étudiants avec le milieu universitaire et la société dont ils sont partie prenante. Sur la base des deux notions de confiance et d'acculturation, nous avons défini trois objectifs :

1. identifier et analyser les facteurs personnels qui soutiennent ou freinent leur persévérance, 
2. identifier et analyser les facteurs relatifs au processus général d'acculturation à la société qui soutiennent ou freinent leur persévérance,

3. identifier les effets possibles de la confiance et de l'acculturation sur leurs représentations sociales quant à leur rôle et leur statut.

Nous nous sommes posé la question suivante : en quoi leur vécu universitaire influence-t-il les facteurs de persévérance et d'échec de ces étudiants, en lien avec leur présence et leur situation dans le contexte québécois? Nous avons fait l'hypothèse qu'ils font l'expérience d'un certain niveau d'isolement, tant dans le milieu universitaire que sociétal, ce qui participe d'un processus d'acculturation posant lui-même un frein à leur cheminement universitaire. Notre recherche permet de documenter ce cheminement en portant une attention particulière à l'adaptation institutionnelle et sociétale ainsi qu'à l'impact de l'effectivité du projet migratoire.

Dans cet article, nous n'exploitons que les données obtenues auprès des étudiants résidents permanents inscrits à I'Université de Montréal et aux deux écoles qui y sont affiliées, soit HEC Montréal et Polytechnique, et qui forment ensemble Campus Montréal. Au total, 416 étudiants permanents ont répondu au questionnaire en ligne et 44 à des groupes de discussion. La mise en commun des données obtenues sur les questions relatives aux relations sociales entretenues par ces étudiants au sein des institutions universitaires liées à Campus Montréal nous a permis d'identifier certaines problématiques récurrentes parmi la population étudiée. Bien que notre étude se soit déroulée en contexte québécois, les résultats présentés peuvent servir à éclairer des situations et des pratiques spécifiques aux étudiants résidents permanents présents dans d’autres contextes nationaux.

Dans un premier temps, on constate peu de contacts soutenus entre les étudiants résidents permanents interrogés et les étudiants issus du groupe majoritaire. En revisitant les problèmes rencontrés par une majorité de répondants, on constate que les plus saillants se situent au niveau des interactions et des relations intergroupes, ce qui affecte directement le niveau de confiance des étudiants résidents permanents à l'égard de la société et de leur projet migratoire. Ces problèmes sont les suivants :

1. Les répondants considèrent que leur origine ethnique et religieuse constitue un critère dans le fonctionnement des relations quotidiennes, ce qui les désavantage, notamment lors des travaux d'équipe;

2. L'intégration culturelle avec les non-immigrants est rendue difficile par la barrière linguistique. Cette dernière relève d'une connaissance minimale du français par les étudiants résidents permanents, mais également par une adaptation difficile à l'accent québécois et par une réticence sentie de la part des natifs à socialiser avec eux;

3. Plusieurs étudiants résidents permanents ont mentionné qu'une certaine discrimination, un racisme latent ou encore des préjugés avaient cours lors des travaux d'équipe. Des répondants considèrent positivement le fait que certains professeurs forment eux-mêmes des équipes de travail multiculturelles.

Ces données permettent de mieux comprendre la subtilité des barrières auxquelles cette population fait face. Rappelons que la majorité de ces étudiants ont fait l'objet d'une sélection rigoureuse de la part des instances gouvernementales. Cette sélection, dont les principes de base sont l'accueil d'individus dont les compétences sont susceptibles de leur permettre de rejoindre rapidement le marché du travail et de contribuer activement au développement socioéconomique de leur société d'accueil, devrait justement conduire à une intégration rapide. Indépendamment de la question du retour aux études - un défi en soi -, les étudiants résidents permanents que nous avons interrogés ont une image plutôt négative de leurs relations avec le groupe majoritaire au Québec. Outre leur incidence au niveau des relations entre individus, ces interactions entre minoritaires et majoritaires agissent par leur nature même comme autant d'effets démobilisants à une échelle plus large. Peu enclins à participer à la vie associative de leur institution et de la société, les étudiants résidents permanents semblent développer une perception plutôt négative de leur intégration professionnelle à moyen et long termes. Loin d'être envisagées comme un tremplin vers une mobilité socio-économique, les études en contexte migratoire pourraient agir en sens inverse, c'est-à-dire être jugées comme constituant une anticipation des problèmes qui attendent ces 
étudiants une fois leurs études terminées. Perçues généralement comme un moyen de se mettre à niveau pour être capable de rivaliser avec les natifs, les études effectuées par les étudiants résidents permanents offriraientelles en fait un simple avant-goût des barrières systémiques et autres qui attendent ces professionnels sur le marché de l'emploi? Si cette hypothèse se vérifiait, alors ce retour aux études n'atteindrait pas ses objectifs, en particulier celui d'atténuer les iniquités en matière d'opportunités d'intégration socio-économiques. 